\title{
ITERATIVE SCHEMES FOR TRIEQUILIBRIUM-LIKE PROBLEMS
}

\section{MISBAH IRAM BLOACH, MUHAMMAD ASLAM NOOR* AND KHALIDA INAYAT NOOR}

Department of Mathematics, COMSATS University Islamabad, Islamabad, Pakistan

*Corresponding author: noormaslam@gmail.com

\begin{abstract}
In this work, we present a new class of equilibrium problems, termed as triequilibrium-like problems with trifunction in invexity settings. Classical varilunational-like inequalities and equilibrium-like problems can be obtained as specific variants of triequilibrium-like problems. Certain new iterative methods are proposed and examined for the solution of triequilibrium-like problems by using auxiliary principle technique. Convergence analysis of these proposed methods is examined under some mild conditions.
\end{abstract}

\section{INTRODUCTION}

Equilibrium problems theory has shown tremendous potential and great influence in various branches of pure and applied sciences. This theory has shown continuously expanding growth in almost all areas of engineering and mathematical sciences. Equilibrium problems cover a diverse set of applications including hemicariational inequalities, variational inequalities, game theory, Nash equilibrium, variational-like inequalities as special use cases, see [16-26]. Blum and Oettli [5] and Noor and Oettli [12] introduced the present form of equilibrium problems. The classical equilibrium problems theory revolves around the assumption of convexity of the set and objective function .

Recently, the notion of convexity has started expanding to numerous fields showing the capacity for various useful applications. Hanson [8] derived invex functions as a special extension of convex functions. Different

Received June 26 $6^{\text {th }}, 2021$; accepted July 20 th, 2021 ; published August $12^{\text {th }}, 2021$.

2010 Mathematics Subject Classification. 49J40, 90C33.

Key words and phrases. auxiliary principle technique; equilibrium problems; proximal point methods; convergence; variational-like inequalities.

(C)2021 Authors retain the copyrights of their papers, and all open access articles are distributed under the terms of the Creative Commons Attribution License. 
results were presented to make this fact noticeable that what holds for convex functions in mathematical programming also holds for a generalized class of functions known as invex functions. Ben-Israel and Mond [3] and Weir and Jeyakumar [29] works led to preinvex functions as another generalized class of convex functions. Weir and Mond [30] has shown in their work the interchangeability of preinvex functions with convex functions in optimization problems. Noor [13] proved that the minimum of preinvex on the invex set can be disciplined into variational inequalities, widely known as variational-like inequalities. Variational-like inequalities and equilibrium-like problems, owing to their specialized nature, cannot allow traditional resolvent method, projection method and their prevalent variant forms to propound any iterative methods. To bridge this gap, we resort to a technique named as auxiliary principle, proposed by Glowinski et al. [6]. To solve numerous variational inequalities and equilibrium problems, Noor [14-20,26] employed the technique of auxiliary principle to propose various iterative methods.

Motivated by the recent research in this field, we introduce another class of equilibrium problems in invexity settings, termed triequilibrium-like problems. We analyze the proximal point methods for triequilibriumlike problems and prove convergence of the generated sequences to a solution of the problem, assuming the existence of solutions and rather mild jointly pseudo monotonicity property of the trifunction $\Psi(., .,$.$) and$

operator $\Upsilon$. In the end, in combination with the Bregman function, we use the auxiliary principle technique again to propose and evaluate some three-step iterative methods for solving triequilibrium-like problems. The convergence of iterative methods is considered by using the partially relaxed strongly- $\eta$ monotonicity and by assuming that the nonlinear term $\Phi(.,$.$) of the triequilibrium-like problems is a skew-symmetric$ function. Since variational inequalities and equilibrium problems are special variants of triequilibrium-like problems, so our results will continue to hold o true for these problems. The techniques and ideas of this paper may be a strating point for further research activities in these dynamic fields.

\section{Preliminaries}

Let $\tilde{H}$ be a real Hilbert space. The inner product and norm on $\tilde{H}$ are denoted by $\langle.,$.$\rangle and \|$. \| respectively. Let $\mathbb{K}_{\hbar}$ be a nonempty invex set in $\tilde{H}$. Let $\Im: \mathbb{K}_{\hbar} \rightarrow \Re$ and $\hbar(.,):. \tilde{H} \times \tilde{H} \rightarrow \tilde{H}$ are continuous functions. Before going on to our main results, first, we recall the following well-known results and concepts.

Definition 2.1. A nonempty set $\mathbb{K}_{\hbar}$ in $\tilde{H}$ is said to be an invex set, if there exists a bifunction $\hbar(.,$.$) , such$ that

$$
\xi+\tau \hbar(\zeta, \xi) \in \mathbb{K}_{\hbar}, \forall \xi, \zeta \in \mathbb{K}_{\hbar}, \tau \in[0,1]
$$

If the set $\mathbb{K}_{\hbar}$ is invex at each $\xi \in \mathbb{K}_{\hbar}$, then $\mathbb{K}_{\hbar}$ is also called $\hbar$-connected set. 
Definition 2.2. A function $\Im: \mathbb{K}_{\hbar} \rightarrow \Re$ is said to be a preinvex function, if there exists a bifunction $\hbar(.,$.$) ,$ such that

$$
\Im(\xi+\tau \hbar(\zeta, \xi)) \leq(1-\tau) \Im(\xi)+\tau \Im(\zeta), \forall \xi, \zeta \in \mathbb{K}_{\hbar}, \tau \in[0,1] .
$$

The function $\Im: \mathbb{K}_{\hbar} \rightarrow \Re$ is said to be preconcave if and only if $-\Im$ is preinvex.

Definition 2.3. A differentiable function $\Im: \mathbb{K}_{\hbar} \rightarrow \Re$ is said to be an invex function, if there exists a bifunction $\hbar(.,$.$) , such that$

$$
\Im(\zeta)-\Im(\xi) \geq\left\langle\Im^{\prime}(\xi), \hbar(\zeta, \xi)\right\rangle, \forall \xi, \zeta \in \mathbb{K}_{\hbar}, \tau \in[0,1]
$$

where $\Im^{\prime}(\xi)$ is the differential of $\Im$ at $\xi$. From above definitions, it is clear that the differentiable preinvex functions are the invex functions but the converse is not true, see [30].

Definition 2.4. A function $\Psi: \mathbb{K}_{\hbar} \rightarrow \Re$ is said to be a strongly preinvex function with respect to a bifunction $\hbar(.,$.$) , if there exists a constant v>0$, such that

$$
\Psi(\xi+\tau \hbar(\zeta, \xi)) \leq(1-\tau) \Psi(\xi)+\tau \Psi(\zeta)-v \tau(1-\tau)\|\hbar(\zeta, \xi)\|^{2}, \forall \xi, \zeta \in \mathbb{K}_{\hbar}, \tau \in[0,1] .
$$

We note that, if $\hbar(\zeta, \xi)=\zeta-\xi$, the invex set $\mathbb{K}_{\hbar}$ reduces to the convex set $\mathbb{K}$ and preinvex functions and strongly preinvex functions reduce to convex functions and strongly convex functions.

Assumption 2.1. The bifunction $\hbar(.,):. \tilde{H} \times \tilde{H} \rightarrow \Re$ satisfies the following condition

$$
\hbar\left(\xi+\tau_{1}(\zeta-\xi), \xi+\tau_{2}(\zeta-\xi)\right)=\left(\tau_{1}-\tau_{2}\right) \hbar(\zeta, \xi), \forall \xi, \zeta \in \tilde{H}
$$

For $\tau_{1}=0,1$ and $\tau_{2}=\tau$, we get Condition C of Mohan and Neogy [10],

$$
\begin{aligned}
& (i) \hbar(\xi, \xi+\tau(\zeta-\xi))=-\tau \hbar(\zeta, \xi) \\
& (i i) \hbar(\zeta, \xi+\tau(\zeta-\xi))=(1-\tau) \hbar(\zeta, \xi), \forall \xi, \zeta \in \tilde{H}
\end{aligned}
$$

Assumption 2.2. The bifunction $\hbar(.,):. \tilde{H} \times \tilde{H} \rightarrow \Re$ satisfies the following condition

$$
\hbar(\xi, \zeta)=\hbar(\xi, \mathfrak{z})+\hbar(\mathfrak{z}, \zeta), \forall \xi, \zeta, \mathfrak{z} \in \tilde{H}
$$

From Assumption (2.2), we get

$$
\begin{aligned}
& \text { (i) } \hbar(\xi, \xi)=0, \forall \xi \in \tilde{H}, \\
& (i i) \hbar(\xi, \zeta)=-\hbar(\zeta, \xi), \forall \xi, \zeta \in \tilde{H} .
\end{aligned}
$$

Assumption (2.2) has a significant role in studying the existence of unique solution of variational-like inequalities and it is also used to suggest and analyze some iterative methods for various classes of variational-like inequalities and equilibrium-like problems. 
Definition 2.5. The bifunction $\hbar(.,):. \tilde{H} \times \tilde{H} \rightarrow \tilde{H}$ is said to be:

(a) Strongly monotone, if there exists a constant $\sigma>0$, such that

$$
\langle\hbar(\zeta, \xi), \zeta-\xi\rangle \geq \sigma\|\zeta-\xi\|^{2}, \forall \xi, \zeta \in \tilde{H}
$$

(b) Lipschitz continuous, if there exists a constant $\delta>0$, such that

$$
\|\hbar(\zeta, \xi)\| \leq \delta\|\zeta-\xi\|, \forall \xi, \zeta \in \tilde{H}
$$

From (a) and (b), it is observed that $\sigma \leq \delta$.

Remark 2.1. If $\hbar(\zeta, \xi)=\Upsilon(\zeta)-\Upsilon(\xi)$, then strong monotonicity and Lipschitz continuity of bifunction $\hbar(.,$.$) reduces to the strong monotonicity and Lipschitz continuity of the nonlinear operator \Upsilon$.

Lemma 2.1. For all $\xi, \zeta \in \tilde{H}$, we have

$$
\begin{aligned}
& \text { (i) } 2\langle\xi, \zeta\rangle=\|\xi+\zeta\|^{2}-\|\xi\|^{2}-\|\zeta\|^{2} \\
& \text { (ii) } 2\langle\xi, \zeta\rangle \leq\|\xi\|^{2}+\|\zeta\|^{2} .
\end{aligned}
$$

Given an operator $\Upsilon: \tilde{H} \rightarrow \Re$, a continuous trifunction $\Psi(., . .):. \tilde{H} \times \tilde{H} \times \tilde{H} \rightarrow \Re$ and a continuous bifunction $\Phi(.,):. \tilde{H} \times \tilde{H} \rightarrow \Re \cup\{+\infty\}$, consider the problem of finding $\xi \in \tilde{H}$, such that

$$
\Psi(\xi, \Upsilon(\xi), \hbar(\zeta, \xi))+\Phi(\zeta, \xi)-\Phi(\xi, \xi)+v\|\hbar(\zeta, \xi)\|^{2} \geq 0, \forall \zeta \in \tilde{H}
$$

The problem (2.1) is called a triequilibrium-like problem. For the physical and mathematical formulation of the problem (2.1), see [1-19,21-24,27-31] and the references therein. Now we discuss some special variants of problem (2.1).

For $\Psi(\xi, \Upsilon(\xi), \hbar(\zeta, \xi))=\langle\xi, \Upsilon(\xi), \hbar(\zeta, \xi)\rangle$, problem (2.1) reduces to a trivariational-like inequality:

$$
\langle\xi, \Upsilon(\xi), \hbar(\zeta, \xi)\rangle+\Phi(\zeta, \xi)-\Phi(\xi, \xi)+v\|\hbar(\zeta, \xi)\|^{2} \geq 0, \forall \zeta \in \tilde{H}
$$

For $\hbar(\zeta, \xi)=\zeta-\xi$, the invex set reduces to the convex set and the problem (2.1) reduces to:

$$
\Psi(\xi, \Upsilon(\xi), \zeta-\xi)+\Phi(\zeta, \xi)-\Phi(\xi, \xi)+v\|\zeta-\xi\|^{2} \geq 0, \forall \zeta \in \tilde{H}
$$

which is called a triequilibrium problem and appears to be new.

Also the trivariational-like inequality (2.2) reduces to:

$$
\langle\xi, \Upsilon(\xi), \zeta-\xi\rangle+\Phi(\zeta, \xi)-\Phi(\xi, \xi)+v\|\zeta-\xi\|^{2} \geq 0, \forall \zeta \in \tilde{H}
$$

The problem (2.4) is called a trivariational inequality. Briefly, choosing proper $\Psi(., .,),. \hbar(.,$.$) and spaces,$ one can get many known and unknown equilibrium problems and special variants of (2.1), see [17-19,21-24]. 
Definition 2.6. The operator $\Upsilon: \tilde{H} \rightarrow \Re$ and the trifunction $\Psi(., .,$.$) are said to be:$

(i) jointly pseudo $\hbar-$ monotone, if

$$
\begin{aligned}
& \Psi(\xi, \Upsilon(\xi), \hbar(\zeta, \xi))+\Phi(\zeta, \xi)-\Phi(\xi, \xi)+v\|\hbar(\zeta, \xi)\|^{2} \geq 0, \\
\Longrightarrow & \\
& \Psi(\zeta, \Upsilon(\zeta), \hbar(\zeta, \xi))+\Phi(\zeta, \xi)-\Phi(\xi, \xi)-v\|\hbar(\zeta, \xi)\|^{2} \geq 0, \forall \xi, \zeta \in \tilde{H},
\end{aligned}
$$

(ii) partially relaxed strongly jointly $\hbar-$ monotone, if a constant $\alpha>0$ exists, such that

$$
\Psi(\xi, \Upsilon(\xi), \hbar(\zeta, \mathfrak{z}))+\Psi(\zeta, \Upsilon(\zeta), \hbar(\mathfrak{z}, \zeta)) \leq \alpha\|\hbar(\mathfrak{z}, \xi)\|^{2}, \forall \xi, \zeta, \mathfrak{z} \in \tilde{H}
$$

(iii) jointly $\hbar-$ monotone, if

$$
\Psi(\xi, \Upsilon(\xi), \hbar(\zeta, \xi))+\Psi(\zeta, \Upsilon(\zeta), \hbar(\xi, \zeta)) \leq 0, \forall \xi, \zeta \in \tilde{H}
$$

(iv) jointly hemicontinuous, if the mapping

$$
\Psi(\xi+\tau \hbar(\xi, \zeta), \Upsilon(\xi+\tau \hbar(\xi, \zeta)), \hbar(\zeta, \xi)) \forall \xi, \zeta \in \tilde{H}, \tau \in[0,1]
$$

is continuous. We noted that, for $\mathfrak{z}=\xi$, partially relaxed strongly jointly $\hbar$ - monotonicity reduces to jointly $\hbar-$ monotonicity.

Definition 2.7. The bifunction $\Phi(.,):. \tilde{H} \times \tilde{H} \rightarrow \Re \cup\{+\infty\}$ is said to be skew symmetric if and only if,

$$
\Phi(\xi, \xi)-\Phi(\xi, \zeta)-\Phi(\zeta, \xi)+\Phi(\zeta, \zeta) \geq 0, \forall \xi, \zeta \in \tilde{H}
$$

If the bifunction $\Phi(.,$.$) is skew symmetric and bilinear then,$

$$
\Phi(\xi, \xi)-\Phi(\xi, \zeta)-\Phi(\zeta, \xi)+\Phi(\zeta, \zeta)=\Phi(\xi-\zeta, \xi-\zeta) \geq 0, \forall \xi, \zeta \in \tilde{H}
$$

which shows that the bifunction $\Phi(.,$.$) is nonnegative.$

\section{ITERATIVE METHODS}

In this section, we derive new iterative methods for a triequilibrium-like problem (2.1) via auxiliary principle technique.

Given $\xi \in \tilde{H}$ satisfying (2.1), consider an auxiliary problem of finding $\varsigma \in \tilde{H}$, such that

$$
\begin{array}{r}
\rho \Psi(\varsigma, \Upsilon(\varsigma), \hbar(\zeta, \varsigma))+\langle\varsigma-\xi, \zeta-\varsigma\rangle+\rho \Phi(\zeta, \varsigma) \\
-\rho \Phi(\varsigma, \varsigma)+\rho v\|\hbar(\zeta, \varsigma)\|^{2} \geq 0, \forall \zeta \in \tilde{H},
\end{array}
$$

which is called an auxiliary triequilibrium-like problem. If $\varsigma=\xi$, then $\varsigma$ is the solution of (2.1). Due to this perception, we consider and evaluate the present iterative methods for solving problem (2.1). 
Algorithm 3.1. Given $\xi_{0} \in \tilde{H}$, find the approximate solution $\xi_{n+1}$, by the iterative scheme

$$
\begin{array}{r}
\rho \Psi\left(\xi_{n+1}, \Upsilon\left(\xi_{n+1}\right), \hbar\left(\zeta, \xi_{n+1}\right)\right)+\left\langle\xi_{n+1}-\xi_{n}, \zeta-\xi_{n+1}\right\rangle+\rho \Phi\left(\zeta, \xi_{n+1}\right) \\
-\rho \Phi\left(\xi_{n+1}, \xi_{n+1}\right)+\rho v\left\|\hbar\left(\zeta, \xi_{n+1}\right)\right\|^{2} \geq 0, \forall \zeta \in \tilde{H}
\end{array}
$$

which is termed as proximal point method for solving triequilibrium-like problems (2.1).

For $\Psi(\xi, \Upsilon(\xi), \hbar(\zeta, \xi))=\langle\xi, \Upsilon(\xi), \hbar(\zeta, \xi)\rangle$, Algorithm (3.1) reduces to:

Algorithm 3.2. Given $\xi_{0} \in \tilde{H}$, find the approximate solution $\xi_{n+1}$, by the iterative scheme

$$
\begin{array}{r}
\rho\left\langle\xi_{n+1}, \Upsilon\left(\xi_{n+1}\right), \hbar\left(\zeta, \xi_{n+1}\right)\right\rangle+\left\langle\xi_{n+1}-\xi_{n}, \zeta-\xi_{n+1}\right\rangle+\rho \Phi\left(\zeta, \xi_{n+1}\right) \\
-\rho \Phi\left(\xi_{n+1}, \xi_{n+1}\right)+\rho v\left\|\hbar\left(\zeta, \xi_{n+1}\right)\right\|^{2} \geq 0, \forall \zeta \in \tilde{H} .
\end{array}
$$

For $\hbar(\zeta, \xi)=\zeta-\xi$, Algorithm (3.1) reduces to:

Algorithm 3.3. Given $\xi_{0} \in \tilde{H}$, find the approximate solution $\xi_{n+1}$, by the iterative scheme

$$
\begin{array}{r}
\rho \Psi\left(\xi_{n+1}, \Upsilon\left(\xi_{n+1}\right), \zeta-\xi_{n+1}\right)+\left\langle\xi_{n+1}-\xi_{n}, \zeta-\xi_{n+1}\right\rangle+\rho \Phi\left(\zeta, \xi_{n+1}\right) \\
-\rho \Phi\left(\xi_{n+1}, \xi_{n+1}\right)+\rho v\left\|\zeta-\xi_{n+1}\right\|^{2} \geq 0, \forall \zeta \in \tilde{H}
\end{array}
$$

For $\hbar(\zeta, \xi)=\zeta-\xi$, Algorithm (3.2) reduces to:

Algorithm 3.4. For the given $\xi_{0} \in \tilde{H}$, find the approximate solution $\xi_{n+1}$ by the iterative scheme

$$
\begin{array}{r}
\rho\left\langle\xi_{n+1}, \Upsilon\left(\xi_{n+1}\right), \zeta-\xi_{n+1}\right\rangle+\left\langle\xi_{n+1}-\xi_{n}, \zeta-\xi_{n+1}\right\rangle+\rho \Phi\left(\zeta, \xi_{n+1}\right) \\
-\rho \Phi\left(\xi_{n+1}, \xi_{n+1}\right)+\rho v\left\|\zeta-\xi_{n+1}\right\|^{2} \geq 0, \forall \zeta \in \tilde{H}
\end{array}
$$

Convergence analysis of Algorithm (3.1) is considered, by adopting the technique of Noor [22].

Theorem 3.1. Let $\bar{\xi} \in \tilde{H}$ be the solution of (2.1) and let $\xi_{n+1}$ be the approximate solution attained by Algorithm (3.1). If the trifunction $\Psi(., .,$.$) and \Upsilon$ are jointly pseudo $\hbar$-monotone and $\Phi(.,$.$) is skew symmetric,$ then

$$
\left\|\xi_{n+1}-\bar{\xi}\right\|^{2} \leq\left\|\xi_{n}-\bar{\xi}\right\|^{2}-\left\|\xi_{n+1}-\xi_{n}\right\|^{2}
$$

Proof: Let $\bar{\xi} \in \tilde{H}$ be the solution of (2.1). Then, using the jointly pseudo $\hbar-$ monotonicity of $\Psi(., .,$.$) ,$ we get

$$
\Psi(\zeta, \Upsilon(\zeta), \hbar(\zeta, \bar{\xi}))+\Phi(\zeta, \bar{\xi})-\Phi(\bar{\xi}, \bar{\xi})-v\|\hbar(\zeta, \bar{\xi})\|^{2} \geq 0, \forall \zeta \in \tilde{H}
$$


Now taking $\zeta=\xi_{n+1}$ in (3.7), we have

$$
\begin{array}{r}
\rho \Psi\left(\xi_{n+1}, \Upsilon\left(\xi_{n+1}\right), \hbar\left(\xi_{n+1}, \bar{\xi}\right)\right)+\rho \Phi\left(\xi_{n+1}, \bar{\xi}\right)-\rho \Phi(\bar{\xi}, \bar{\xi}) \\
-\rho v\left\|\hbar\left(\xi_{n+1}, \bar{\xi}\right)\right\|^{2} \geq 0
\end{array}
$$

Now taking $\zeta=\bar{\xi}$ in $(3.2)$, we get

$$
\begin{array}{r}
\rho \Psi\left(\xi_{n+1}, \Upsilon\left(\xi_{n+1}\right), \hbar\left(\bar{\xi}, \xi_{n+1}\right)\right)+\left\langle\xi_{n+1}-\xi_{n}, \bar{\xi}-\xi_{n+1}\right\rangle+\rho \Phi\left(\bar{\xi}, \xi_{n+1}\right) \\
-\rho \Phi\left(\xi_{n+1}, \xi_{n+1}\right)+\rho v\left\|\hbar\left(\bar{\xi}, \xi_{n+1}\right)\right\|^{2} \geq 0
\end{array}
$$

which can be written as

$$
\begin{aligned}
\left\langle\xi_{n+1}-\xi_{n}, \bar{\xi}-\xi_{n+1}\right\rangle & \geq-\rho \Psi\left(\xi_{n+1}, \Upsilon\left(\xi_{n+1}\right), \hbar\left(\bar{\xi}, \xi_{n+1}\right)\right)-\rho v\left\|\hbar\left(\bar{\xi}, \xi_{n+1}\right)\right\|^{2} \\
& +\rho \Phi\left(\xi_{n+1}, \xi_{n+1}\right)-\rho \Phi\left(\bar{\xi}, \xi_{n+1}\right) \\
& \geq \rho\left\{\Phi(\bar{\xi}, \bar{\xi})-\Phi\left(\bar{\xi}, \xi_{n+1}\right)\right. \\
& \left.-\Phi\left(\xi_{n+1}, \bar{\xi}\right)+\Phi\left(\xi_{n+1}, \xi_{n+1}\right)\right\} \\
& \geq 0
\end{aligned}
$$

where we used (3.8) and skew symmetry of $\Phi(.,$.$) . Now by using Lemma (2.1), we get$

$$
\left\|\xi_{n+1}-\bar{\xi}\right\|^{2} \leq\left\|\xi_{n}-\bar{\xi}\right\|^{2}-\left\|\xi_{n+1}-\xi_{n}\right\|^{2}
$$

the desired result.

Theorem 3.2. Let a finite-dimensional space $\tilde{H}$, and an approximate solution $\left\{\xi_{n+1}\right\}$, given by Algorithm (3.1). If $\bar{\xi} \in \tilde{H}$ be the solution of (2.1), then $\lim _{n \rightarrow \infty} \xi_{n}=\bar{\xi}$.

Proof : Let $\bar{\xi} \in \tilde{H}$ be the solution of (2.1). From (3.6), it follows that $\left\|\bar{\xi}-\xi_{n}\right\|$ is monotonically decreasing and hence $\left\{\xi_{n}\right\}$ is bounded. Furthermore from (3.6), we have

$$
\sum_{n=0}^{\infty}\left\|\xi_{n+1}-\xi_{n}\right\|^{2} \leq\left\|\xi_{0}-\bar{\xi}\right\|^{2}
$$

From above, we get

$$
\lim _{n \longrightarrow \infty}\left\|\xi_{n+1}-\xi_{n}\right\|=0
$$

A subsequence $\left\{\xi_{n_{j}}\right\}$ of bounded sequence $\left\{\xi_{n}\right\}$ is convergent to the cluster point $\hat{\xi} \in \tilde{H}$ of $\left\{\xi_{n}\right\}$. Replacing $\xi_{n}$ by $\xi_{n_{j}}$ and letting $n_{j} \longrightarrow \infty$ in (3.2) and using (3.11), we have

$$
\Psi(\hat{\xi}, \Upsilon(\hat{\xi}), \hbar(\zeta, \hat{\xi}))+\Phi(\zeta, \hat{\xi})-\Phi(\hat{\xi}, \hat{\xi})+v\|\hbar(\zeta, \hat{\xi})\|^{2} \geq 0, \forall \zeta \in \tilde{H}
$$


which implies that $\hat{\xi}$ solves $(2.1)$ and

$$
\left\|\xi_{n+1}-\hat{\xi}\right\|^{2} \leq\left\|\xi_{n}-\hat{\xi}\right\|^{2}
$$

From above it follows that, the sequence $\left\{\xi_{n}\right\}$ has a unique cluster point $\hat{\xi}$ and

$$
\lim _{n \longrightarrow \infty} \xi_{n}=\hat{\xi}
$$

which is our required result.

For the implementation of the proximal methods, to determine the approximate solution implicitly is a tricky problem. To deal with this issue, we propose another iterative method for solving (2.1).

For a given $\xi \in \tilde{H}$ satisfying (2.1), we consider the auxiliary problem of finding $\varsigma \in \tilde{H}$, such that

$$
\begin{array}{r}
\rho \Psi(\xi, \Upsilon(\xi), \hbar(\zeta, \varsigma))+\langle\varsigma-\xi, \zeta-\varsigma\rangle+\rho \Phi(\zeta, \varsigma)-\rho \Phi(\varsigma, \varsigma) \\
+\rho v\|\hbar(\zeta, \varsigma)\|^{2} \geq 0, \forall \zeta \in \tilde{H},
\end{array}
$$

where constant $\rho>0$. Notably the problem (3.1) is not decomposable and not the interpretation of an optimization problem whereas problem (3.12) is almost the optimization problem. For $\varsigma=\xi$, $\varsigma$ is the solution of (2.1). By using this observation we suggest and analyze the present iterative methods for solving problem (2.1).

Algorithm 3.5. Given $\xi_{0} \in \tilde{H}$, find the approximate solution $\xi_{n+1}$, by the iterative scheme

$$
\begin{array}{r}
\rho \Psi\left(\xi_{n}, \Upsilon\left(\xi_{n}\right), \hbar\left(\zeta, \xi_{n+1}\right)\right)+\left\langle\xi_{n+1}-\xi_{n}, \zeta-\xi_{n+1}\right\rangle+\rho \Phi\left(\zeta, \xi_{n+1}\right) \\
-\rho \Phi\left(\xi_{n+1}, \xi_{n+1}\right)+\rho v\left\|\hbar\left(\zeta, \xi_{n+1}\right)\right\|^{2} \geq 0, \forall \zeta \in \tilde{H}
\end{array}
$$

For $\Psi(\xi, \Upsilon(\xi), \hbar(\zeta, \xi))=\langle\xi, \Upsilon(\xi), \hbar(\zeta, \xi)\rangle$, Algorithm (3.5) reduces to:

Algorithm 3.6. Given $\xi_{0} \in \tilde{H}$, find the approximate solution $\xi_{n+1}$, by the iterative scheme

$$
\begin{array}{r}
\rho\left\langle\xi_{n}, \Upsilon\left(\xi_{n}\right), \hbar\left(\zeta, \xi_{n+1}\right)\right\rangle+\left\langle\xi_{n+1}-\xi_{n}, \zeta-\xi_{n+1}\right\rangle+\rho \Phi\left(\zeta, \xi_{n+1}\right) \\
-\rho \Phi\left(\xi_{n+1}, \xi_{n+1}\right)+\rho v\left\|\hbar\left(\zeta, \xi_{n+1}\right)\right\|^{2} \geq 0, \forall \zeta \in \tilde{H}
\end{array}
$$

For $\hbar(\zeta, \xi)=\zeta-\xi$, Algorithm (3.5) reduces to:

Algorithm 3.7. Given $\xi_{0} \in \tilde{H}$, find the approximate solution $\xi_{n+1}$, by the iterative scheme

$$
\begin{array}{r}
\rho \Psi\left(\xi_{n}, \Upsilon\left(\xi_{n}\right), \zeta-\xi_{n+1}\right)+\left\langle\xi_{n+1}-\xi_{n}, \zeta-\xi_{n+1}\right\rangle+\rho \Phi\left(\zeta, \xi_{n+1}\right) \\
-\rho \Phi\left(\xi_{n+1}, \xi_{n+1}\right)+\rho v\left\|\zeta-\xi_{n+1}\right\|^{2} \geq 0, \forall \zeta \in \tilde{H} .
\end{array}
$$

For $\hbar(\zeta, \xi)=\zeta-\xi$, Algorithm (3.6) reduces to: 
Algorithm 3.8. Given $\xi_{0} \in \tilde{H}$, find the approximate solution $\xi_{n+1}$, by the iterative scheme

$$
\begin{array}{r}
\rho\left\langle\xi_{n}, \Upsilon\left(\xi_{n}\right), \zeta-\xi_{n+1}\right\rangle+\left\langle\xi_{n+1}-\xi_{n}, \zeta-\xi_{n+1}\right\rangle+\rho \Phi\left(\zeta, \xi_{n+1}\right) \\
-\rho \Phi\left(\xi_{n+1}, \xi_{n+1}\right)+\rho v\left\|\zeta-\xi_{n+1}\right\|^{2} \geq 0, \forall \zeta \in \tilde{H}
\end{array}
$$

Equivalently, many unique and familiar algorithms for solution of a triequilibrium-like problems can be obtained for best fitting operators and spaces.

We consider the convergence analysis of Algorithm (3.5) by using the condition of partially relaxed strongly $\hbar-$ monotonicity.

Theorem 3.3. Let $\bar{\xi} \in \tilde{H}$ be the solution of (2.1) and let $\xi_{n+1}$ be the approximate solution attained by Algorithm (3.5). If the trifunction $\Psi(., .,$.$) and \Upsilon$ be partially relaxed strongly jointly $\hbar-$ monotone with constant $\alpha>0$ and $\hbar(.,):. \tilde{H} \times \tilde{H} \rightarrow \tilde{H}$ be Lipchitz-continuous with constant $\delta>0$, then

$$
\left\{1-4 \rho v \delta^{2}\right\}\left\|\xi_{n+1}-\bar{\xi}\right\|^{2} \leq\left\|\xi_{n}-\bar{\xi}\right\|^{2}-\left\{1-2 \rho \alpha \delta^{2}\right\}\left\|\xi_{n+1}-\xi_{n}\right\|^{2}
$$

Proof: Let $\bar{\xi} \in \tilde{H}$ be the solution of (2.1), then

$$
\Psi(\bar{\xi}, \Upsilon(\bar{\xi}), \hbar(\zeta, \bar{\xi}))+\Phi(\zeta, \bar{\xi})-\Phi(\bar{\xi}, \bar{\xi})+v\|\hbar(\zeta, \bar{\xi})\|^{2} \geq 0, \forall \zeta \in \tilde{H}
$$

Now taking $\zeta=\xi_{n+1}$ in (3.18), we have

$$
\Psi\left(\bar{\xi}, \Upsilon(\bar{\xi}), \hbar\left(\xi_{n+1}, \bar{\xi}\right)\right)+\Phi\left(\xi_{n+1}, \bar{\xi}\right)-\Phi(\bar{\xi}, \bar{\xi})+v\left\|\hbar\left(\xi_{n+1}, \bar{\xi}\right)\right\|^{2} \geq 0
$$

Now taking $\zeta=\bar{\xi}$ in $(3.13)$, we get

$$
\begin{array}{r}
\rho \Psi\left(\xi_{n}, \Upsilon\left(\xi_{n}\right), \hbar\left(\bar{\xi}, \xi_{n+1}\right)\right)+\left\langle\xi_{n+1}-\xi_{n}, \bar{\xi}-\xi_{n+1}\right\rangle+\rho \Phi\left(\bar{\xi}, \xi_{n+1}\right) \\
-\rho \Phi\left(\xi_{n+1}, \xi_{n+1}\right)+\rho v\left\|\hbar\left(\bar{\xi}, \xi_{n+1}\right)\right\|^{2} \geq 0, \forall \zeta \in \tilde{H}
\end{array}
$$

By adding (3.19) and (3.20), we get

$$
\begin{aligned}
\left\langle\xi_{n+1}-\xi_{n}, \bar{\xi}-\xi_{n+1}\right\rangle & \geq-\rho\left\{\Psi\left(\xi_{n}, \Upsilon\left(\xi_{n}\right), \hbar\left(\bar{\xi}, \xi_{n+1}\right)\right)+\Psi\left(\bar{\xi}, \Upsilon(\bar{\xi}), \hbar\left(\xi_{n+1}, \bar{\xi}\right)\right)\right\} \\
& -2 \rho v\left\|\hbar\left(\xi_{n+1}, \bar{\xi}\right)\right\|^{2}+\rho\left\{\Phi(\bar{\xi}, \bar{\xi})-\Phi\left(\bar{\xi}, \xi_{n+1}\right)\right. \\
& \left.-\Phi\left(\xi_{n+1}, \bar{\xi}\right)+\Phi\left(\xi_{n+1}, \xi_{n+1}\right)\right\} \\
& \geq-\rho \alpha\left\|\hbar\left(\xi_{n}, \xi_{n+1}\right)\right\|^{2}-2 \rho v\left\|\hbar\left(\xi_{n+1}, \bar{\xi}\right)\right\|^{2} \\
& \geq-\rho \alpha \delta^{2}\left\|\xi_{n+1}-\xi_{n}\right\|^{2}-2 \rho v \delta^{2}\left\|\xi_{n+1}-\bar{\xi}\right\|^{2}
\end{aligned}
$$

where we used the partially relaxed strongly jointly $\hbar-$ monotonicity of $\Psi(., .,$.$) with constant \alpha>0$, the skew symmetry of $\Phi(.,$.$) and the Lipschitz continuity of \hbar(.,$.$) . Now by using Lemma (2.1), we get$

$$
2\left\langle\xi_{n+1}-\xi_{n}, \bar{\xi}-\xi_{n+1}\right\rangle=\left\|\xi_{n}-\bar{\xi}\right\|^{2}-\left\|\xi_{n+1}-\bar{\xi}\right\|^{2}-\left\|\xi_{n+1}-\xi_{n}\right\|^{2}
$$


By combining (3.21) and (3.22), we have

$$
\left\{1-4 \rho v \delta^{2}\right\}\left\|\xi_{n+1}-\bar{\xi}\right\|^{2} \leq\left\|\xi_{n}-\bar{\xi}\right\|^{2}-\left\{1-2 \rho \alpha \delta^{2}\right\}\left\|\xi_{n+1}-\xi_{n}\right\|^{2},
$$

which is the required (3.17). To inquire the convergence of Algorithm (3.5), we can employ the similar procedure of Theorem (3.2).

Now we consider the inertial proximal method for the solution of problem (2.1) by using auxiliary principle technique.

For a given $\xi \in \tilde{H}$ satisfying (2.1), we consider the auxiliary problem of finding $\varsigma \in \tilde{H}$, such that

$$
\begin{array}{r}
\rho \Psi(\varsigma, \Upsilon(\varsigma), \hbar(\zeta, \varsigma))+\langle\varsigma-\xi-\gamma(\xi-\xi), \zeta-\varsigma\rangle+\rho \Phi(\zeta, \varsigma) \\
-\rho \Phi(\varsigma, \varsigma)+\rho v\|\hbar(\zeta, \varsigma)\|^{2} \geq 0, \forall \zeta \in \tilde{H}
\end{array}
$$

where $\rho>0$ and $\gamma>0$ are constants.

The problem (3.23) is called the auxiliary triequilibrium-like problem. If $\varsigma=\xi$, then $\varsigma$ is the solution of (2.1). This observation motivates us to suggest and analyze the below-mentioned inertial proximal method for (2.1).

Algorithm 3.9. Given $\xi_{0} \in \tilde{H}$, find the approximate solution $\xi_{n+1}$, by the iterative scheme

$$
\begin{array}{r}
\rho \Psi\left(\xi_{n+1}, \Upsilon\left(\xi_{n+1}\right), \hbar\left(\zeta, \xi_{n+1}\right)\right)+\left\langle\xi_{n+1}-\xi_{n}-\gamma_{n}\left(\xi_{n}-\xi_{n-1}\right), \zeta-\xi_{n+1}\right\rangle \\
\quad+\rho \Phi\left(\zeta, \xi_{n+1}\right)-\rho \Phi\left(\xi_{n+1}, \xi_{n+1}\right)+\rho v\left\|\hbar\left(\zeta, \xi_{n+1}\right)\right\|^{2} \geq 0, \forall \zeta \in \tilde{H}
\end{array}
$$

For $\gamma_{n}=0$, Algorithm (3.9) reduces to the Algorithm (3.1). This shows that the proximal methods are included in inertial proximal methods as special variants, see $[2,14]$.

We now consider the convergence criteria of Algorithm (3.9) by adopting the technique of Alvarez [1] and Noor [15].

Theorem 3.4. Let $\bar{\xi} \in \tilde{H}$ be the solution of (2.1) and $\xi_{n+1}$ be the approximate solution attained by Algorithm (3.9). If the trifunction $\Psi(., .,$.$) and \Upsilon$ are jointly pseudo $\hbar-$ monotone and the bifunction $\hbar(.,$.$) is Lipschitz$ continuous with constant $\delta>0$, then

$$
\begin{aligned}
\left\|\xi_{n+1}-\bar{\xi}\right\|^{2} & \leq\left\|\xi_{n}-\bar{\xi}\right\|^{2}-\left\|\xi_{n+1}-\xi_{n}-\gamma_{n}\left(\xi_{n}-\xi_{n-1}\right)\right\|^{2} \\
& +\gamma_{n}\left\{\left\|\xi_{n}-\bar{\xi}\right\|^{2}-\left\|\bar{\xi}-\xi_{n-1}\right\|^{2}+2\left\|\xi_{n}-\xi_{n-1}\right\|^{2}\right\}
\end{aligned}
$$

Proof: Let $\bar{\xi} \in \tilde{H}$ be a solution of (2.1), then by using the jointly pseudo $\hbar$ - monotonicity of $\Psi(., .,$. and $\Upsilon$, we get

$$
\Psi(\zeta, \Upsilon(\zeta), \hbar(\zeta, \bar{\xi}))+\Phi(\zeta, \bar{\xi})-\Phi(\bar{\xi}, \bar{\xi})-v\|\hbar(\zeta, \bar{\xi})\|^{2} \geq 0, \forall \zeta \in \tilde{H}
$$


Now taking $\zeta=\xi_{n+1}$ in (3.26), we have

$$
\begin{array}{r}
\rho \Psi\left(\xi_{n+1}, \Upsilon\left(\xi_{n+1}\right), \hbar\left(\xi_{n+1}, \bar{\xi}\right)\right)+\rho \Phi\left(\xi_{n+1}, \bar{\xi}\right)-\rho \Phi(\bar{\xi}, \bar{\xi}) \\
-\rho v\left\|\hbar\left(\xi_{n+1}, \bar{\xi}\right)\right\|^{2} \geq 0 .
\end{array}
$$

Now taking $\zeta=\bar{\xi}$ in $(3.24)$, we get

$$
\begin{array}{r}
\rho \Psi\left(\xi_{n+1}, \Upsilon\left(\xi_{n+1}\right), \hbar\left(\bar{\xi}, \xi_{n+1}\right)\right)+\left\langle\xi_{n+1}-\xi_{n}-\gamma_{n}\left(\xi_{n}-\xi_{n-1}\right), \bar{\xi}-\xi_{n+1}\right\rangle \\
+\rho \Phi\left(\bar{\xi}, \xi_{n+1}\right)-\rho \Phi\left(\xi_{n+1}, \xi_{n+1}\right)+\rho v\left\|\hbar\left(\bar{\xi}, \xi_{n+1}\right)\right\|^{2} \geq 0
\end{array}
$$

by combining (3.27) and (3.28) and by using skew symmetry of $\Phi(.,$.$) , we get$

$$
\begin{aligned}
\left\langle\xi_{n+1}-\xi_{n}-\gamma_{n}\left(\xi_{n}-\xi_{n-1}\right), \bar{\xi}-\xi_{n+1}\right\rangle & \geq-\rho \Psi\left(\xi_{n+1}, \Upsilon\left(\xi_{n+1}\right), \hbar\left(\bar{\xi}, \xi_{n+1}\right)\right)+\rho \Phi\left(\xi_{n+1}, \xi_{n+1}\right) \\
& -\rho \Phi\left(\bar{\xi}, \xi_{n+1}\right)-\rho v\left\|\hbar\left(\bar{\xi}, \xi_{n+1}\right)\right\|^{2} \\
& \geq \rho\left\{\Phi(\bar{\xi}, \bar{\xi})-\Phi\left(\bar{\xi}, \xi_{n+1}\right)\right. \\
& \left.-\Phi\left(\xi_{n+1}, \bar{\xi}\right)+\Phi\left(\xi_{n+1}, \xi_{n+1}\right)\right\} \\
& \geq 0 .
\end{aligned}
$$

We can write (3.29) in the form

$$
\left\langle\xi_{n+1}-\xi_{n}, \bar{\xi}-\xi_{n+1}\right\rangle \geq \gamma_{n}\left\{\left\langle\xi_{n}-\xi_{n-1}, \bar{\xi}-\xi_{n}\right\rangle+\left\langle\xi_{n}-\xi_{n-1}, \xi_{n}-\xi_{n+1}\right\rangle\right\}
$$

Now by using Lemma (2.1), we get

$$
\begin{gathered}
2\left\langle\xi_{n+1}-\xi_{n}, \bar{\xi}-\xi_{n+1}\right\rangle=\left\|\xi_{n}-\bar{\xi}\right\|^{2}-\left\|\xi_{n+1}-\bar{\xi}\right\|^{2}-\left\|\xi_{n+1}-\xi_{n}\right\|^{2} \\
2 \gamma_{n}\left\langle\xi_{n}-\xi_{n-1}, \bar{\xi}-\xi_{n}\right\rangle=\gamma_{n}\left\{\left\|\bar{\xi}-\xi_{n-1}\right\|^{2}-\left\|\xi_{n}-\bar{\xi}\right\|^{2}-\left\|\xi_{n}-\xi_{n-1}\right\|^{2}\right\} .
\end{gathered}
$$

By substituting (3.31) and (3.32) in (3.30) and rearranging terms, we get (3.25), the desired result .

Theorem 3.5. Let a finite-dimensional space $\tilde{H}$, and an approximate solution $\left\{\xi_{n+1}\right\}$, given by Algorithm (3.9). If $\bar{\xi} \in H$ be the solution of (2.1), then there exists $\gamma \in(0,1)$, such that $0 \leq \gamma_{n} \leq \gamma, \forall n \in N$ and the following condition holds

$$
\sum_{n=1}^{\infty} \gamma_{n}\left\|\xi_{n}-\xi_{n-1}\right\|^{2} \leq \infty
$$

then $\lim _{n \longrightarrow \infty} \xi_{n}=\bar{\xi}$. 
Proof : Let $\bar{\xi} \in \tilde{H}$ be a solution of (2.1). We first examine the case where $\gamma_{n}=0$, which corresponds to the standard proximal method. In this case from (3.25), as a result the sequence $\left\{\left\|\bar{\xi}-\xi_{n}\right\|\right\}$ is monotonically decreasing and $\left\{\xi_{n}\right\}$ is bounded. Furthermore from (3.25), we get

$$
\sum_{n=0}^{\infty}\left\|\xi_{n+1}-\xi_{n}\right\|^{2} \leq\left\|\xi_{0}-\bar{\xi}\right\|^{2}
$$

from above, we get

$$
\lim _{n \longrightarrow \infty}\left\|\xi_{n+1}-\xi_{n}\right\|=0
$$

A subsequence $\left\{\xi_{n_{j}}\right\}$ of bounded sequence $\left\{\xi_{n}\right\}$ is convergent to the cluster point $\hat{\xi} \in \tilde{H}$ of $\left\{\xi_{n}\right\}$. Replacing $\xi_{n}$ by $\xi_{n_{j}}$ and letting $n_{j} \longrightarrow \infty$ in (3.24) and using (3.33), we have

$$
\Psi(\hat{\xi}, \Upsilon(\hat{\xi}), \hbar(\zeta, \hat{\xi}))+\Phi(\zeta)-\Phi(\hat{\xi})+v\|\hbar(\zeta, \hat{\xi})\|^{2} \geq 0, \forall \zeta \in \tilde{H}
$$

implies that, $\hat{\xi}$ solves the triequilibrium-like problem (2.1) and

$$
\left\|\xi_{n+1}-\hat{\xi}\right\|^{2} \leq\left\|\xi_{n}-\hat{\xi}\right\|^{2}
$$

Hence, the sequence $\left\{\xi_{n}\right\}$ has exactly one cluster point $\hat{\xi}$ and

$$
\lim _{n \longrightarrow \infty} \xi_{n}=\hat{\xi}
$$

which is our required result. Now we consider the case $\gamma_{n}>0$. From (3.25) and using the technique of Alvarez [1,2] and Noor [15], we have

$$
\begin{aligned}
& \sum_{n=1}^{\infty}\left\|\xi_{n+1}-\xi_{n}-\gamma_{n}\left(\xi_{n}-\xi_{n-1}\right)\right\|^{2} \\
& \leq\left\|\xi_{0}-\bar{\xi}\right\|^{2}+\sum_{n=1}^{\infty} \gamma\left(\left\|\xi_{n}-\bar{\xi}\right\|^{2}+2\left\|\xi_{n}-\xi_{n-1}\right\|^{2}\right) \\
& \leq \infty,
\end{aligned}
$$

which implies that

$$
\lim _{n \longrightarrow \infty}\left\|\xi_{n+1}-\xi_{n}-\gamma_{n}\left(\xi_{n}-\xi_{n-1}\right)\right\|^{2}=0 .
$$

By repeating the above arguments, rest of the proof runs as in the case $\gamma_{n}=0$, one can easily find the required result, that is $\lim _{n \longrightarrow \infty} \xi_{n}=\hat{\xi}$.

For a given $\xi \in \tilde{H}$ satisfying problem (2.1), we consider the auxiliary problem of finding $\varsigma \in \tilde{H}$, such that

$$
\begin{array}{r}
\rho \Psi(\xi, \Upsilon(\xi), \hbar(\zeta, \varsigma))+\left\langle\mathbb{E}^{\prime}(\varsigma)-\mathbb{E}^{\prime}(\xi), \hbar(\zeta, \varsigma)\right\rangle+\rho \Phi(\zeta, \varsigma) \\
-\rho \Phi(\varsigma, \varsigma)+\rho v\|\hbar(\zeta, \varsigma)\|^{2} \geq 0, \forall \zeta \in \tilde{H}
\end{array}
$$


which is called an auxiliary triequilibrium-like problem associated with the problem (2.1). Problem (3.34) has a unique solution because $\mathbb{E}^{\prime}($.$) is the differential of strongly preinvex function. Noted that, if \varsigma=\xi$, then $\varsigma$ is the solution of problem (2.1). This observation leads us to suggest and examine the following methods for problem (2.1).

Algorithm 3.10. Given $\xi_{0} \in \tilde{H}$, find the approximate solution $\xi_{n+1}$, by the iterative scheme

$$
\begin{array}{r}
\rho \Psi\left(\varsigma_{n}, \Upsilon\left(\varsigma_{n}\right), \hbar\left(\zeta, \xi_{n+1}\right)\right)+\left\langle\mathbb{E}^{\prime}\left(\xi_{n+1}\right)-\mathbb{E}^{\prime}\left(\varsigma_{n}\right), \hbar\left(\zeta, \xi_{n+1}\right)\right\rangle+ \\
\rho \Phi\left(\zeta, \xi_{n+1}\right)-\rho \Phi\left(\xi_{n+1}, \xi_{n+1}\right)+\rho v\left\|\hbar\left(\zeta, \xi_{n+1}\right)\right\|^{2} \geq 0, \forall \zeta \in \tilde{H} \\
\lambda \Psi\left(y_{n}, \Upsilon\left(y_{n}\right), \hbar\left(\zeta, \varsigma_{n}\right)\right)+\left\langle\mathbb{E}^{\prime}\left(\varsigma_{n}\right)-\mathbb{E}^{\prime}\left(y_{n}\right), \hbar\left(\zeta, \varsigma_{n}\right)\right\rangle+\lambda \Phi\left(\zeta, \varsigma_{n}\right) \\
-\lambda \Phi\left(\varsigma_{n}, \varsigma_{n}\right)+\lambda v\left\|\hbar\left(\zeta, \varsigma_{n}\right)\right\|^{2} \geq 0, \forall \zeta \in \tilde{H}, \\
\mu \Psi\left(\xi_{n}, \Upsilon\left(\xi_{n}\right), \hbar\left(\zeta, y_{n}\right)\right)+\left\langle\mathbb{E}^{\prime}\left(y_{n}\right)-\mathbb{E}^{\prime}\left(\xi_{n}\right), \hbar\left(\zeta, y_{n}\right)\right\rangle+\mu \Phi\left(\zeta, y_{n}\right) \\
-\mu \Phi\left(y_{n}, y_{n}\right)+v \mu\left\|\hbar\left(\zeta, y_{n}\right)\right\|^{2} \geq 0, \forall \zeta \in \tilde{H}
\end{array}
$$

which is the three-step iterative algorithm for solving the triequilibrium - like problem (2.1), where $\rho>0, \lambda>0$ and $\mu>0$ are constants.

For $\mu=0$, Algorithm (3.10) reduces to the two-step iterative algorithm for (2.1).

Algorithm 3.11. Given $\xi_{0} \in \tilde{H}$, find the approximate solution $\xi_{n+1}$, by the iterative scheme

$$
\begin{array}{r}
\rho \Psi\left(\varsigma_{n}, \Upsilon\left(\varsigma_{n}\right), \hbar\left(\zeta, \xi_{n+1}\right)\right)+\left\langle\mathbb{E}^{\prime}\left(\xi_{n+1}\right)-\mathbb{E}^{\prime}\left(\varsigma_{n}\right), \hbar\left(\zeta, \xi_{n+1}\right)\right\rangle+ \\
\rho \Phi\left(\zeta, \xi_{n+1}\right)-\rho \Phi\left(\xi_{n+1}, \xi_{n+1}\right)+\rho v\left\|\hbar\left(\zeta, \xi_{n+1}\right)\right\|^{2} \geq 0, \forall \zeta \in \tilde{H}, \\
\lambda \Psi\left(\xi_{n}, \Upsilon\left(\xi_{n}\right), \hbar\left(\zeta, \varsigma_{n}\right)\right)+\left\langle\mathbb{E}^{\prime}\left(\varsigma_{n}\right)-\mathbb{E}^{\prime}\left(\xi_{n}\right), \hbar\left(\zeta, \varsigma_{n}\right)\right\rangle+\lambda \Phi\left(\zeta, \varsigma_{n}\right) \\
-\lambda \Phi\left(\varsigma_{n}, \varsigma_{n}\right)+\lambda v\left\|\hbar\left(\zeta, \varsigma_{n}\right)\right\|^{2} \geq 0, \quad \forall \zeta \in \tilde{H},
\end{array}
$$

For $\mu=0, \lambda=0$, Algorithm (3.10) as follows.

Algorithm 3.12. Given $\xi_{0} \in \tilde{H}$, find the approximate solution $\xi_{n+1}$, by the iterative scheme

$$
\begin{aligned}
& \rho \Psi\left(\xi_{n}, \Upsilon\left(\xi_{n}\right), \hbar\left(\zeta, \xi_{n+1}\right)\right)+\left\langle\mathbb{E}^{\prime}\left(\xi_{n+1}\right)-\mathbb{E}^{\prime}\left(\xi_{n}\right), \hbar\left(\zeta, \xi_{n+1}\right)\right\rangle+ \\
& \rho \Phi\left(\zeta, \xi_{n+1}\right)-\rho \Phi\left(\xi_{n+1}, \xi_{n+1}\right)+\rho v\left\|\hbar\left(\zeta, \xi_{n+1}\right)\right\|^{2} \geq 0, \forall \zeta \in \tilde{H}
\end{aligned}
$$

Now we consider the convergence criteria of Algorithm (3.10) by using the technique of Noor [16]. 
Theorem 3.6. Let $\mathbb{E}(\xi)$ be a differentiable strongly preinvex function with modulus $\beta>0$. Let the trifunction $\Psi(., .,$.$) and operator \Upsilon: \tilde{H} \rightarrow \Re$ be partially relaxed strongly-jointly $\hbar-$ monotone with constant $\alpha>0$. If Assumption (2.2) holds and there exists the constants $0<\rho<\frac{\beta}{\alpha}, 0<\lambda<\frac{\beta}{\alpha}$, and $0<\mu<\frac{\beta}{\alpha}$, then an approximate solution $\xi_{n+1}$ given by Algorithm (3.10) converges to solution $\xi \in \tilde{H}$ of (2.1).

Proof: Let $\xi \in \tilde{H}$ be a solution of (2.1), then

$$
\begin{array}{r}
\rho \Psi(\xi, \Upsilon(\xi), \hbar(\zeta, \xi))+\rho \Phi(\zeta, \xi)-\rho \Phi(\xi, \xi) \\
+\rho v\|\hbar(\zeta, \xi)\|^{2} \geq 0, \forall \zeta \in \tilde{H}
\end{array}
$$

$$
\begin{array}{r}
\lambda \Psi(\xi, \Upsilon(\xi), \hbar(\zeta, \xi))+\lambda \Phi(\zeta, \xi)-\lambda \Phi(\xi, \xi) \\
+\lambda v\|\hbar(\zeta, \xi)\|^{2} \geq 0, \quad \forall \zeta \in \tilde{H}
\end{array}
$$

$$
\begin{array}{r}
\mu \Psi(\xi, \Upsilon(\xi), \hbar(\zeta, \xi))+\mu \Phi(\zeta, \xi)-\mu \Phi(\xi, \xi) \\
+\mu v\|\hbar(\zeta, \xi)\|^{2} \geq 0, \forall \zeta \in \tilde{H}
\end{array}
$$

Taking $\zeta=\xi_{n+1}$ in (3.38), we get

$$
\rho \Psi\left(\xi, \Upsilon(\xi), \hbar\left(\xi_{n+1}, \xi\right)\right)+\rho \Phi\left(\xi_{n+1}, \xi\right)-\rho \Phi(\xi, \xi)+\rho v\left\|\hbar\left(\xi_{n+1}, \xi\right)\right\|^{2} \geq 0
$$

Now taking $\zeta=\xi$ in (3.35), we get

$$
\begin{aligned}
& \rho \Psi\left(\varsigma_{n}, \Upsilon\left(\varsigma_{n}\right), \hbar\left(\xi, \xi_{n+1}\right)\right)+\left\langle\mathbb{E}^{\prime}\left(\xi_{n+1}\right)-\mathbb{E}^{\prime}\left(\varsigma_{n}\right), \hbar\left(\xi, \xi_{n+1}\right)\right\rangle \\
& +\rho \Phi\left(\xi, \xi_{n+1}\right)-\rho \Phi\left(\xi_{n+1}, \xi_{n+1}\right)+\rho v\left\|\hbar\left(\xi, \xi_{n+1}\right)\right\|^{2} \geq 0 .
\end{aligned}
$$

Consider the function

$$
\begin{aligned}
\mathfrak{B}(\mathfrak{z}) & =\mathbb{E}(\xi)-\mathbb{E}(\mathfrak{z})-\left\langle\mathbb{E}^{\prime}(\mathfrak{z}), \hbar(\xi, \mathfrak{z})\right\rangle \\
& \geq \beta\|\hbar(\xi, \mathfrak{z})\|^{2}, \forall \xi, \mathfrak{z} \in \tilde{H} .
\end{aligned}
$$


Since $\mathbb{E}($.$) is strongly preinvex function. By combining (3.41), (3.42) and (3.43) and using Assumption (2.2),$ we get

$$
\begin{aligned}
\mathfrak{B}\left(\varsigma_{n}\right)-\mathfrak{B}\left(\xi_{n+1}\right) & =\mathbb{E}\left(\xi_{n+1}\right)-\mathbb{E}\left(\varsigma_{n}\right)-\left\langle\mathbb{E}^{\prime}\left(\varsigma_{n}\right), \hbar\left(\xi, \varsigma_{n}\right)\right\rangle+\left\langle\mathbb{E}^{\prime}\left(\xi_{n+1}\right), \hbar\left(\xi, \xi_{n+1}\right)\right\rangle \\
& =\mathbb{E}\left(\xi_{n+1}\right)-\mathbb{E}\left(\varsigma_{n}\right)+\left\langle\mathbb{E}^{\prime}\left(\xi_{n+1}\right)-\mathbb{E}^{\prime}\left(\varsigma_{n}\right), \hbar\left(\xi, \xi_{n+1}\right)\right\rangle \\
& -\left\langle\mathbb{E}^{\prime}\left(\varsigma_{n}\right), \hbar\left(\xi_{n+1}, \varsigma_{n}\right)\right\rangle \\
& \geq \beta\left\|\hbar\left(\xi_{n+1}, \varsigma_{n}\right)\right\|^{2}+\left\langle\mathbb{E}^{\prime}\left(\xi_{n+1}\right)-\mathbb{E}^{\prime}\left(\varsigma_{n}\right), \hbar\left(\xi, \xi_{n+1}\right)\right\rangle \\
& \geq \beta\left\|\hbar\left(\xi_{n+1}, \varsigma_{n}\right)\right\|^{2}-\rho\left[\Psi\left(\varsigma_{n}, \Upsilon\left(\varsigma_{n}\right), \hbar\left(\xi, \xi_{n+1}\right)\right)\right. \\
& \left.+\Psi\left(\xi, \Upsilon(\xi), \hbar\left(\xi_{n+1}, \xi\right)\right)\right]+\rho\left[\Phi\left(\xi_{n+1}, \xi_{n+1}\right)-\Phi\left(\xi, \xi_{n+1}\right)\right. \\
& \left.-\Phi\left(\xi_{n+1}, \xi\right)+\Phi(\xi, \xi)\right]+\rho v_{1}\left\|\hbar\left(\xi, \xi_{n+1}\right)\right\|^{2} \\
& \geq(\beta-\rho \alpha)\left\|\hbar\left(\xi_{n+1}, \varsigma_{n}\right)\right\|^{2}+\rho v_{1}\left\|\hbar\left(\xi, \xi_{n+1}\right)\right\|^{2} \\
& \geq(\beta-\rho \alpha)\left\|\hbar\left(\xi_{n+1}, \varsigma_{n}\right)\right\|^{2}
\end{aligned}
$$

where we used skew symmetry of bifunction $\Phi(.,$.$) and partially relaxed strongly joint \hbar-$ monotonicity of trifunction $\Psi(., .,$.$) and operator \Upsilon$ with constant $\alpha>0$. Similarly by using (3.36), (3.37), (3.39) and (3.40) and repeating the same arguments, we get

$$
\begin{aligned}
& \mathfrak{B}\left(y_{n}\right)-\mathfrak{B}\left(\varsigma_{n}\right) \geq(\beta-\lambda \alpha)\left\|\hbar\left(\varsigma_{n}, y_{n}\right)\right\|^{2}, \\
& \mathfrak{B}\left(\xi_{n}\right)-\mathfrak{B}\left(y_{n}\right) \geq(\beta-v \alpha)\left\|\hbar\left(y_{n}, \xi_{n}\right)\right\|^{2} .
\end{aligned}
$$

If $\xi_{n+1}=\varsigma_{n}=y_{n}=\xi_{n}$, then obviously $\xi_{n}$ is the solution of problem(2.1).

Otherwise for $0<\rho<\frac{\beta}{\alpha}, 0<\lambda<\frac{\beta}{\alpha}$ and $0<\mu<\frac{\beta}{\alpha}$ the sequences $\mathfrak{B}\left(\varsigma_{n}\right)-\mathfrak{B}\left(\xi_{n+1}\right), \mathfrak{B}\left(y_{n}\right)-\mathfrak{B}\left(\varsigma_{n}\right)$ and $\mathfrak{B}\left(\xi_{n}\right)-\mathfrak{B}\left(y_{n}\right)$ are non negative, and we must have

$$
\begin{array}{r}
\lim _{n \longrightarrow \infty}\left\|\hbar\left(\xi_{n+1}, \varsigma_{n}\right)\right\|=0, \\
\lim _{n \longrightarrow \infty}\left\|\hbar\left(\varsigma_{n}, y_{n}\right)\right\|=0, \\
\lim _{n \longrightarrow \infty}\left\|\hbar\left(y_{n}, \xi_{n}\right)\right\|=0 .
\end{array}
$$

So,

$$
\begin{array}{r}
\lim _{n \longrightarrow \infty}\left\|\hbar\left(\xi_{n+1}, \xi_{n}\right)\right\|=\lim _{n \longrightarrow \infty}\left\|\hbar\left(\xi_{n+1}, \varsigma_{n}\right)\right\| \\
+\lim _{n \longrightarrow \infty}\left\|\hbar\left(\varsigma_{n}, y_{n}\right)\right\|+\lim _{n \longrightarrow \infty}\left\|\hbar\left(y_{n}, \xi_{n}\right)\right\|=0 .
\end{array}
$$

Zhu and Marcotte [31] technique is employed to show that the entire sequence $\left\{\xi_{n}\right\}$ is convergent to the cluster point $\bar{\xi}$, which satisfies the triequilibrium-like problem(2.1). 


\section{Conclusion}

We have investigated another class of equilibrium problems, termed as triequlibrium-like problems. Several new iterative methods for the solution of this newly class have been proposed. Convergence of these iterative methods was tested by using only partially relaxed strongly jointly $\hbar-$ monotonicity or jointly pseudo $\hbar-$ monotonicity of the operator $\Upsilon$ and the trifunction $\Psi$. Further exploration is needed to actualize these algorithms which will be very fascinating applications for new specialists.

Conflicts of Interest: The author(s) declare that there are no conflicts of interest regarding the publication of this paper.

\section{REFERENCES}

[1] F. Alvarez, On the minimization property of a second order dissipative system in Hilbert space, SIAM J. Control Optim. 38(2000), 1102-1119.

[2] F. Alvarez and H. Attouch, An inertial proximal method for maximal monotone operators via discretization of a nonlinear oscillator damping, Set-Valued Anal. 9(2001), 3-11.

[3] A. Ben-Israel and B. Mond, What is invexity? J. Austral. Math. Soc. Ser. B. 28 (1986), 1-9.

[4] M.I. Bloach and M.A. Noor, Perturbed mixed variational-like inequalities, AIMS Math. 5(3)(2019), 2153-2162.

[5] E. Blum and W. Oettli, From optimization and variational inequalities to equilibrium problems, Math. Student. 63(1994), 123-145.

[6] R. Glowinski, J. L. Lions and R. Tremolieres, Numerical analysis of variational inequalities, North-Holland, Amsterdam, 1981.

[7] F. Giannessi, A. Maugeri and P. M. Pardalos, Equilibrium Problems: Nonsmooth Optimization and Variational inequality Models, Kluwer Academic Publishers, Dordrecht, Holland, 2001.

[8] M. A. Hanson, On sufficiency of the Kuhn-Tucker conditions, J. Math. Anal. Appl. 80(1981), 545-550.

[9] J. L. Lions and G. Stampacchia, Variational inequalities, Comm. Pure Appl. Math. 20(1967), 493-512.

[10] S.R. Mohan and S.K. Neogy, On invex set and preinvex functions, J. Math. Anal. Appl. 189 (1995), 901-908.

[11] B.B. Mohsen, M.A. Noor, K.I. Noor, and M. Postolache, Strongly convex functions of higher order involving bifunction, Mathematics, 7(11)(2019), 1028.

[12] M. A. Noor and W. Oettli, On general nonlinear complementarity problems and quasi-equilibria, Le Mathematiche, 49 (1994), 313-331.

[13] M. A. Noor, Variational-like inequalities, Optimization, 30(1994), 323-330.

[14] M. A. Noor, Proximal methods for mixed quasi variational inequalities, J. Optim. Theory Appl. 115(2002), 447-452.

[15] M. A. Noor, M. Akhter and K. I. Noor, Inertial proximal method for mixed quasi variational inequalities, Nonlinear Funct. Anal. Appl. 8(2003), 489-496.

[16] M. A. Noor, Fundamentals of mixed quasi variational inequalities, Int. J. Pure. Appl. Math. 15(2004), $137-250$.

[17] M. A. Noor and K. I. Noor, On equilibrium problems, Appl. Math. E-Notes. 4(2004), 125-132.

[18] M. A. Noor, Mixed quasi invex equilibrium problems, Int. J. Math. Sci. 57(2004), 3057-3067.

[19] M. A. Noor, Auxiliary principle technique for equilibrium problems, J. Optim. Theory. Appl. 122(2004), 371-386.

[20] M. A. Noor, Some developments in general variational inequalities, Appl. Math. Comput. 251(2004), $199-277$. 
[21] M. A. Noor, Invex equilibrium problems, J. Math. Anal. Appl. 302(2005), 463-475

[22] M. A. Noor, Fundamentals of equilibrium problems, Math. Inequal. Appl. 9 (2006), 529-566.

[23] M. A. Noor, K. I. Noor and V. Gupta, On equilibrium- like problems, Appl. Anal. 86(2007), 807-818.

[24] M. A. Noor, K. I. Noor and M.I Baloch, Auxiliary principle technique for strongly mixed variational-like inequalities, U.P.B. Sci. Bull. Ser. A. 80(2018), 93-100.

[25] M. A. Noor, K. I. Noor, A. Hamdi and E. H. El-shemas, On difference of two monotone operators, Optim. Lett. 3(2009), 329.

[26] M. A. Noor, K. I. Noor and M. Th. Rassias, New trends in general variational inequalitis, Acta Appl. Math. 170(1)(2020), 981-1046.

[27] J. Parida and A. Sen, A variational-like inequality for multifunctions with applications, J. Math. Anal. Appl., 124(1987), $73-81$.

[28] G. Stampacchia, Formes bilineaires coercitives sur les ensembles convexes, C. R. Acad. Sci. Paris, 258(1964), 4413-4416.

[29] T. Weir and V. Jeyakumar, A class of nonconvex functions and mathematical programming, Bull. Austral. Math. Soc. 38(1988), 177-189.

[30] T. Weir and B. Mond, Preinvex functions in multiple objective optimization, J. Math. Anal. Appl. 136(1988), 29-28.

[31] D.L. Zhu and P. Marcotte, Cocoercivity and its role in the convergence of iterative schemes for solving variational inequalities, SIAM J. Optim. 6(3)(1996), 714-726. 